\title{
DENOISING OF LIDAR ECHO SIGNAL BASED ON WAVELET ADAPTIVE THRESHOLD METHOD
}

\author{
Shuhua Long ${ }^{1}$, Guoqing Zhou ${ }^{1}$, Haoyu Wang ${ }^{1}$,Xiang Zhou ${ }^{2,3,}{ }^{*}$,Jinlong Chen ${ }^{1,2}$,Jian Gao ${ }^{1}$ \\ ${ }^{1}$ Guangxi Key Laboratory of Spatial Information and Geomatics, Guilin University of Technology, No. 12 Jian'gan Road, Guilin, \\ Guangxi 541004, China \\ ${ }^{2}$ College of Mechanical and Control Engineering, Guilin University of Technology, No. 12 Jian'gan Road, Guilin, \\ Guangxi 541004, China \\ ${ }^{3}$ School of Microelectronics, Tianjin University, No. 92 Weijin Road, Tianjin 300072, China
}

KEY WORDS: Denoising, Wavelet transform, Gaussian noise, Adaptive thresholding, Signal processing, Signal-to-noise ratio

\begin{abstract}
:
The wavelet threshold method is widely used in signal denoising. However, traditional hard threshold method or soft threshold method is deficient for depending on fixed threshold and instability. In order to achieve efficient denoising of echo signals, an adaptive wavelet threshold denoising method, absorbing the advantages of the hard threshold and the soft threshold, is proposed. Based on the advantages of traditional threshold method, new threshold function is continuous, steerable and flexibly changeable by adjusting two parameters. The threshold function is flexibly changed between the hard threshold and the soft threshold function by two parameter adjustments. According to the Stein unbiased risk estimate (SURE), this new method can determine thresholds adaptively. Adopting different thresholds adaptively at different scales, this method can automatically track noise, which can effectively remove the noise on each scale. Therefore, the problems of noise misjudgement and incomplete denoising can be solved, to some extent, in the process of signal processing. The simulation results of MATLAB show that compared with hard threshold method and soft threshold method, the signalto-noise ratio (SNR) of the proposed de-noising method is increased by nearly $2 \mathrm{~dB}$, and $4 \mathrm{~dB}$ respectively. It is safely to conclude that, when background noise eliminated, the new wavelet adaptive threshold method preserves signal details effectively and enhances the separability of signal characteristics.
\end{abstract}

\section{INTRODUCTION}

Our research team have won a key project of innovation-driven development in Guangxi in 2018 that aims to develop a high precision airborne LiDAR which can survey the seabed in threedimensional. The schematic structure of the instrument is shown in Fig.1. My task is to process the echo signal of LiDAR. Denoising is a crucial issue in signal processing. The details in the observation data of the unsteady signal contain a large amount of feature information (Frei et al., 2007). The characteristics of Lidar signal lidar echo signal is typically nonlinear and unstable. The echo signal detected by airborne laser detection system is not only weak in light intensity, but also has a wide dynamic range, which can generally reach $10^{4} \sim 10^{6}$ (Xu, 2002; Zhou, 2015a). The traditional Fourier transform plays a huge role in the denoising of steady-state signals, but it cannot describe the local information of the unsteady signal. Therefore, it is not suitable for the denoising processing of such signals. The wavelet threshold denoising method is a nonlinear denoising method, which is approximate optimal in the sense of minimum mean square error. At present, wavelet analysis is considered to be the best method for non-stationary signal de-noising.

In 1995, Donoho proposed the wavelet threshold method (Donoho, 1995). By setting an appropriate threshold, the wavelet coefficients of the signal decomposition were modified according to the selected threshold function, and the signal was reconstructed to achieve signal denoising. In the literature, various techniques for adaptive selection of threshold values (Deng et al., 2007; Madeiro et al., 2007). Quan (1998, 1999, 2007) constructs an approximation function of the mean square error function. A lot of research work is done around the optimal threshold, which makes the wavelet threshold filtering method tend to be perfect. In recent years, other scholars have made great efforts to construct the threshold function and obtain the best denoising effect in the sense of mean square error by various methods (Beenamol et al., 2012; Li et al., 2012; Atto et al., 2011; Stefan et al., 2012; Sanam et al., 2013). However, it is not

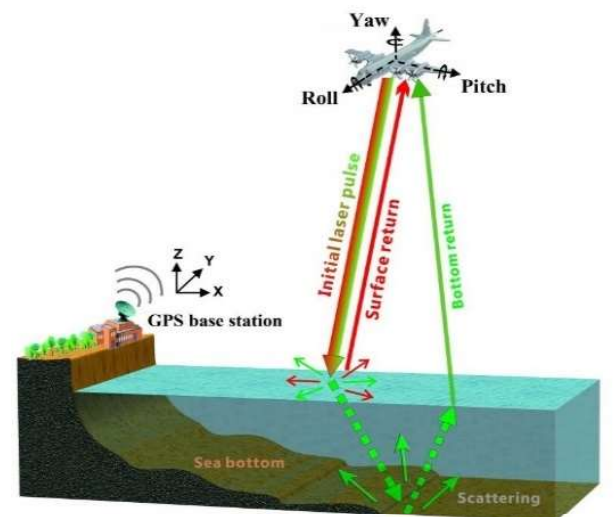

Figure 1. Dual LiDAR working diagram

\footnotetext{
* Corresponding author: Xiang Zhou; E-mail: zqx0711@glut.edu.cn
} 
possible to consider the minimum mean square error as a performance indicator for denoising in some practical applications. For example, the characteristics of signals extracted from low-signal-to-noise LiDAR noisy signals are used for target recognition (Zhou, 2018). The mutation points, signal singularities, often contains key features of the signal. The wavelet transform coefficients of these mutation points are not always greater than the optimal threshold. The ordinary threshold function will weaken or even eliminate the wavelet coefficients of these mutation points seriously, thus losing the key features represented by signal singularity. It even generates enormous difficulties to the subsequent target recognition.

As an ideal signal processing method, the wavelet threshold method still has some shortcomings. Based on utilizing both advantages and disadvantages of the soft and hard threshold methods adequately, this paper constructs a new threshold function, which can overcome the defects of discontinuous and fixed deviation of traditional methods. Furthermore, the selection of threshold is adaptive, which improves the denoising quality of the wavelet threshold method.

\section{WAVELET THRESHOLD DENOISING OF UNSTEADY SIGNALS}

\subsection{Denoising Problem in Singular Feature Extraction of Unsteady Signals}

In the pattern recognition of unsteady noise-containing signals, the singularity of the reserved signals needs to be considered in the process of denoising (Mallat, 1992). This is especially important in low signal-to-noise ratio (SNR), such as lidar signals, where the signal-to-noise ratio is very low (Zhou, $2007,2015 b)$. The real signal is often submerged in the noise, and a large number of singular points representing the target characteristics need to be reserved during the denoising process. As shown in Fig.2, the waveform in Fig.2(a) contains the mutation signal. Fig.2(b) shows the signal waveform after adding Gaussian white noise to the data in Fig.2(a). The signal is basically submerged in the noise. The graph in Fig.2(c) is the effect diagram of the noisy signal denoised by the hard threshold function. The noise is basically filtered out, but the detail signal
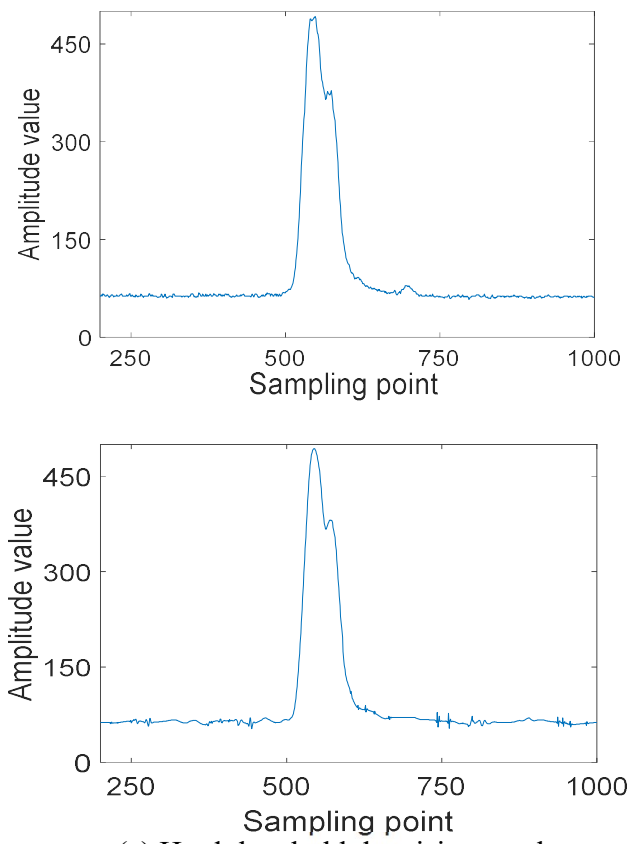

(c) Hard threshold denoising results of the target echo (the rectangular part in the Fig.2(c)) is also smoothed out. Fig.2(d) is a diagram showing the effect of denoising the signal with the soft threshold function. Compared with the hard threshold function filtering, the detail of the signal is better retained, but more noise is also retained. In order to preserve the details of the signal as much as possible during the denoising process, it is necessary to design a new threshold function between the hard threshold function and the soft threshold function.

\subsection{Wavelet Threshold Denoising Model}

Assume the observed data vector $\mathrm{y}=\left[\mathrm{y}_{0}, \mathrm{y}_{1}, \ldots, y_{N-1}\right]^{T}$ given by:

$$
y_{i}=f_{i}+n_{i}, i=0,1, \ldots N-1 .
$$

Where $y_{i}$ is the noisy signal, $f_{i}$ is the useful signal, and $n_{i}$ is the noise (Randhawa, 2018). The noise distribution is Gaussian white noise in Lidar. The useful signal $f_{i}$ in the noisy signal is a low frequency signal, and $n_{i}$ is a high frequency signal. After wavelet transform, the energy is distributed on a few wavelet coefficients, and these wavelet coefficients are significantly distributed on each decomposition scale. The core idea of the wavelet threshold method is to compare the pre-set threshold and the wavelet coefficients after signal decomposition. The wavelet coefficients larger or less than the threshold is reserved or contracted. Then the signal is reconstructed by inverter of wavelet coefficient (Kim et al., 2006). The process of noise reduction by wavelet threshold method is shown in Fig.3.

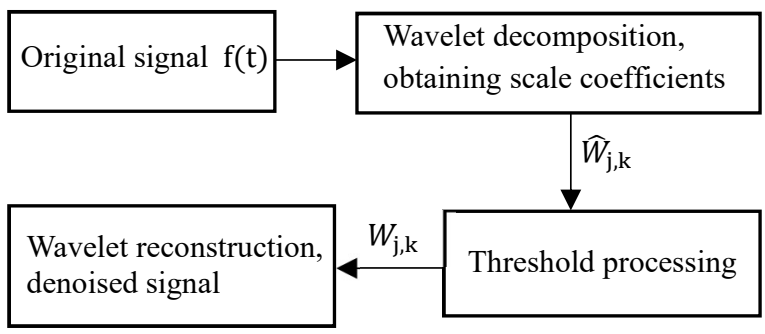

Figure 3. Wavelet threshold method denoising flow chart

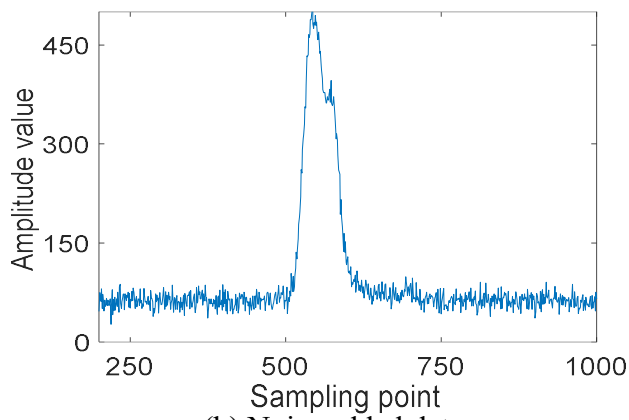

(b) Noise added data

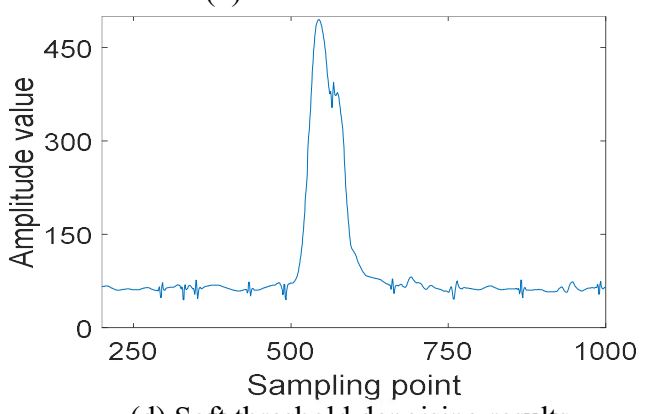

(d) Soft threshold denoising results

Figure 2. Denoising effect of echo signal using traditional threshold function 
It can be seen from the above that the core of the wavelet threshold denoising method is the threshold processing or estimation of the wavelet coefficients. Different wavelet coefficient estimation methods correspond to different wavelet threshold denoising methods. Hard threshold and soft threshold method are traditional methods. As shown in formula (2), the hard threshold function is to decrease wavelet coefficients to 0 when their absolute values are smaller than the threshold, and preserve the wavelet coefficient whose absolute value is larger than the threshold (Nason, 1996). The soft threshold function is to shrink the wavelet coefficients whose absolute values are larger than the threshold, as shown in formula (3) (Jansen, 1997).

$$
\begin{gathered}
W_{j, k}= \begin{cases}W_{j, k},\left|W_{j, k}\right| \geq \lambda \\
0, & \left|W_{j, k}\right|<\lambda\end{cases} \\
W_{j, k}= \begin{cases}\operatorname{sign}\left(W_{j, k}\right) \cdot\left(\left|W_{j, k}\right|-\lambda\right), & \left|W_{j, k}\right| \geq \lambda \\
0, & \left|W_{j, k}\right|<\lambda\end{cases}
\end{gathered}
$$

Where $W_{j, k}$ is the wavelet coefficient, and $\lambda$ is the threshold. The wavelet coefficients processed by the hard threshold function are discontinuous at $\pm \lambda$, leading to poor continuity after transformation. There may be oscillations in the signal via wavelet reconstruction. Meanwhile, there is a certain deviation between the wavelet coefficients and the wavelet coefficients treated by the soft threshold function. As a result, an enormous error exists between the reconstructed signal and the real signal. The error is unavoidable with this method.

\section{ADAPTIVE THRESHOLD METHOD}

\subsection{Constructing a New Threshold Function}

In order to solve the problem of traditional wavelet threshold denoising, it is necessary for the threshold function to be continuous at its threshold point and possesses a high-order derivable property. Thus, the function of selecting the threshold can be realized, and the energy distribution of the decomposed coefficient can be embodied in good condition. Inspired by $\mathrm{Wu}$ et al. (2014), a new threshold function is proposed:

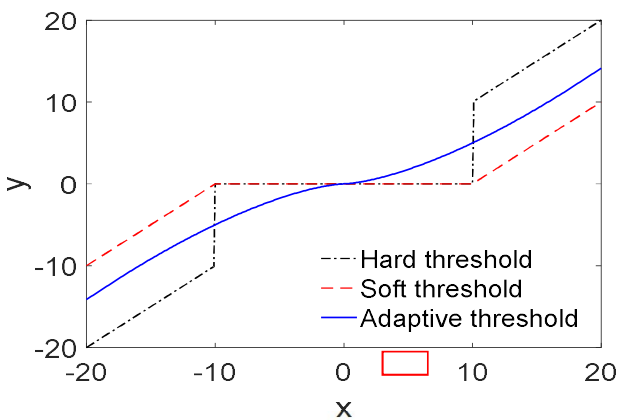

(a) New threshold functions and soft and hard threshold

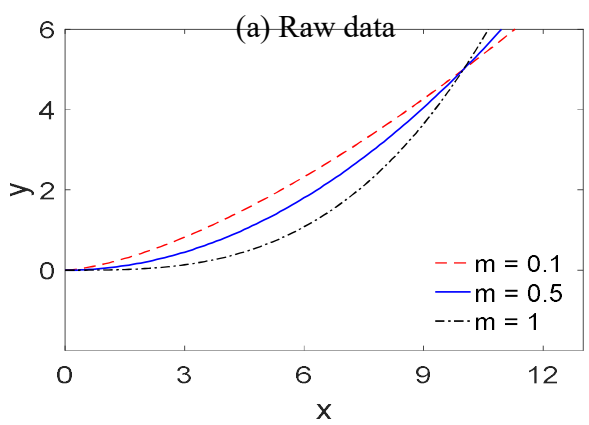

(c) An enlarged view of zone 1 in Figure (b)

$$
\widehat{W}_{j, k}= \begin{cases}x-\operatorname{sign}\left(W_{j, k}\right) \frac{m \lambda^{\alpha}}{2\left|W_{j, k}\right|^{\alpha-1}}, & \left|W_{j, k}\right|>\lambda \\ \operatorname{sign}\left(W_{j, k}\right) \frac{m\left|W_{j, k}\right|^{\alpha+1}}{2 \lambda^{\alpha}}, & \left|W_{j, k}\right| \leq \lambda\end{cases}
$$

Where $W_{j, k}$ and $\widehat{W}_{j, k}$ are wavelet transform coefficients before and after denoising, respectively. Sign( ) is the sign function, and $\lambda$ is the threshold. The newly constructed threshold function is continuous and first-order steerable. The function waveform is shown in Fig.4(a). The parameters $\alpha, \mathrm{m}$ are threshold function adjustment factors. By adjusting their values, the flexibility of the threshold function can be enhanced in practical denoising applications. Ranging from 0 to 1 , the parameter $\mathrm{m}$ determines the shape of the threshold function while $\alpha$ ensures the asymptote. When $\alpha \rightarrow 0$, the new threshold function tends to be a soft threshold function. Oppositely, when $\alpha \rightarrow 1$, the new threshold function tends to be a hard threshold function. Using the new threshold function for wavelet threshold denoising, a smooth transition from noise figure to signal coefficient is accomplished. By observing Fig.4(c) and Fig.4(d), it can be found that the new threshold function can zero, shrink or maintain the wavelet coefficients in different regions, which is different from the soft or hard threshold function. The wavelet transform coefficient in the critical region, a region that can shrink the wavelet coefficient, is composed of signal and noise (Nasri, 2009). The removal ratio of the noise signal or the retention ratio of the signal details can be controlled by adjusting the size of the contraction region, attaining the maximum detail preservation of signal while removing noise. It provides a more realistic initial signal for subsequent target recognition.

In summary, the threshold function proposed in this paper contributes a smooth transition of the wavelet coefficient weakening degree in the critical section. The critical region can be adjusted by $m$. The larger the $m$ is, the closer it is to the hard threshold function, the larger scale contraction of the wavelet coefficients of the critical region. Therefore, it is suitable to process a noisy signal with higher Signal-to-Noise Ratio (SNR)

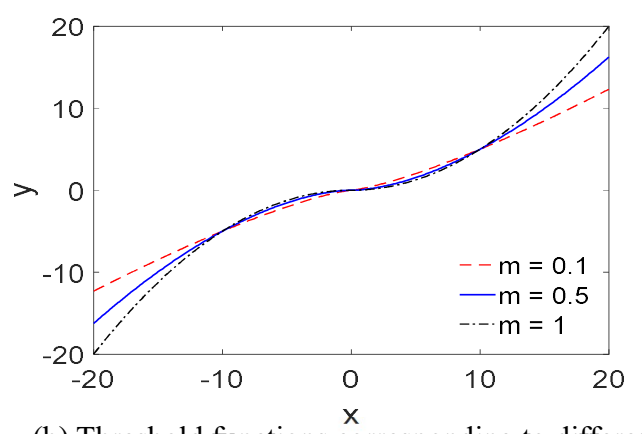

(b) Threshold functions corresponding to different $\mathrm{m}$ values and hard threshold functions

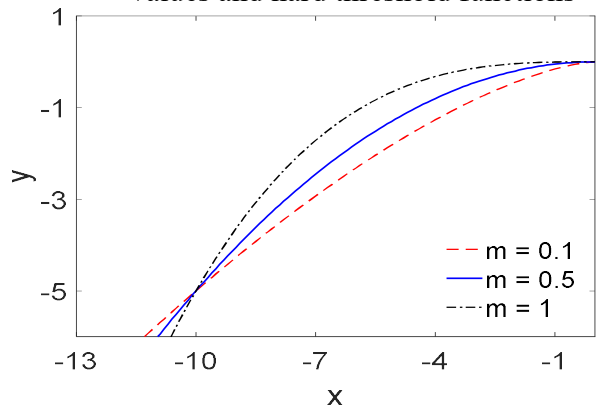

(d) Enlarged view of zone 2 in Figure (b)

Figure 4. New threshold function graph 
when $\mathrm{m}$ is relatively large. Conversely, the smaller the $\mathrm{m}$ value is, the more wavelet coefficients are in the critical region. The signal detail wavelet coefficients can be preserved preferably in the case of shrinking noise coefficients, thereby maintaining the original local singularity of the signal.

\subsection{Adaptive Threshold Determination Method}

The amplitude and density of noise decrease with the increase of scale, while the signal increases with the increase of scale series. If the same threshold is used, some useful information will be removed at a lower scale, leaving some noise at the maximum scale at the same time. The problem we are trying to solve here is how to find the optimal threshold at each level of scale.

In this paper, the optimal threshold in the sense of minimum variance is calculated first, and the minimum variance estimate (MSE) can be estimated by calculating SURE (Stein Unbiased Risk Estimate), that is, the optimal threshold is calculated by SURE. Assuming that the observed signal is $y$, the real signal is $\mathrm{x}$, the noise signal is $\mathrm{n}$, then the mathematical model is as shown in equation (1). The coefficients of these three quantities after wavelet transform are $\hat{u}, \mu$ and $v$, respectively. The corresponding mathematical model is:

$$
\hat{u}=\mu+v
$$

A function is defined:

$$
g(y)=\hat{f}(y)-y
$$

Where $\hat{f}(y)$ is a threshold function, $g=\left[g_{0}, g_{1}, \ldots, g_{N-1}\right]^{T}$ is the mapping function of the $\mathrm{N}$-dimensional vector $y$. The equation can be established as:

$$
\begin{aligned}
E\left[\|g(y)\|^{2}\right] & =E\left[\|\hat{f}(y)-y\|^{2}\right]=E\left[\|\hat{f}(y)-(x+n)\|^{2}\right] \\
& =E\left[\|\hat{f}(y)-x\|^{2}\right]+E[\|n\|]^{2}+0 \\
& =E\left[\|\hat{u}-x\|^{2}\right]+E[\|v\|]^{2}
\end{aligned}
$$

Therefore, when $E\left[\|\hat{u}-x\|^{2}\right]$ takes the minimum value, $E\left[\|g(y)\|^{2}\right]$ also corresponds to the minimum value. Because $g(y)$ is divisible, it can be obtained according to the unbiased estimate of SURE (Stein, 1981):

$$
E\left[\|\hat{f}(y)-y\|^{2}\right]=N+E\left[\|g(y)-x\|^{2}+2 \sum_{i=0}^{N-1} \frac{\partial g_{i}}{\partial y_{i}}\right]
$$

The SURE is an unbiased estimate of equation (8), defined as:

$$
R_{S}(t)=N+[\|g(y)\|]^{2}+2 \sum_{i=0}^{N-1} \frac{\partial g_{i}}{\partial y_{i}}
$$

From equation (7), it can be concluded that the minimum of MSE corresponds to the minimum of the unbiased estimate of SURE. Therefore, when $R_{S}(t)$ in the formula (9) takes the minimum value, the corresponding threshold is the best threshold in the sense of minimum MSE. The gradient function of $\operatorname{Rs}(t)$ is:

$$
\frac{\partial R_{S}(t)}{\partial \lambda}=2 \sum_{i=0}^{N-1} g_{i} \cdot \frac{\partial g_{i}}{\partial \lambda}+2 \sum_{i=0}^{N-1} \frac{\partial^{2} g_{i}}{\partial y_{i} \partial \lambda}
$$

Substituting equation (6) into equation (4) to calculate the partial derivative:

$$
\begin{gathered}
\frac{g_{i}}{\partial \lambda}=\frac{\partial \widehat{W}_{j, k}}{\partial \lambda}=\left\{\begin{array}{l}
-0.5 m a \cdot \operatorname{sign}(y)\left(\frac{\lambda}{|y|}\right)^{a-1},|y|>\lambda \\
-0.5 m a \cdot \operatorname{sign}(y)\left(\frac{|y|}{\lambda}\right)^{a+1},|y| \leq \lambda
\end{array}\right. \\
\frac{\partial^{2} g_{i}}{\partial y_{i} \partial \lambda}=\left\{\begin{array}{l}
0 \quad,|y|>\lambda \\
-0.5 m a(a+1) \cdot \operatorname{sign}(y) \frac{|y|^{a}}{\lambda^{a+1}},|y| \leq \lambda
\end{array}\right.
\end{gathered}
$$

By minimizing $R_{s}^{j}(\lambda)$ on different decomposition scales, the optimal threshold $\lambda^{j}$ on the corresponding scale can be obtained. By using the steepest descent method of the optimization algorithm, the optimal threshold can be acquired.

\section{SIGNAL DENOISING EXPERIMENT SIMULATION}

In the experiment, the lidar echo signal model, named WaLid, proposed by Hani Abdallah et al. is used to simulate the actual observation data by superimposing the zero mean Gaussian white noise, which represents the underwater environmental noise(Abdallah et al., 2012). 500 sets of lidar echo data are processed by the method in this paper to obtain their average values of SNR, root mean square error (RMSE) and local peak relative error (LREPV), respectively. The expressions of the three indicators are (13), (14) and (15) respectively. The (SNR) represents the amount of noise in the signal. The greater the value is, the less the noise content is in the signal.

The RMSE indicates the measurement. The smaller the value is, the higher the measurement reliability is. The LREPV is used to measure the singularity retention of the original signal.

$$
\begin{gathered}
S N R=10 \lg \left(\frac{\sum_{i=1}^{n}\left|W_{j, k}\right|^{2}}{\sum_{i=1}^{n}\left|\widehat{W}_{j, k}-W_{j, k}\right|^{2}}\right) \\
R M S E=\sqrt{\frac{1}{N} \sum_{i=1}^{n}\left|\widehat{W}_{j, k}-W_{j, k}\right|^{2}} \\
L R E P V=\frac{1}{N} \sum_{i=1}^{n} \frac{\left|P_{o i}-P_{d i}\right|}{P_{o i}} \times 100 \%
\end{gathered}
$$

Where $P_{o i}$ is the local peak of the i-th segment of the original signal, and $P_{d i}$ is the corresponding peak of the i-th segment after the signal denoising process

After the echo signal is denoised, it can be seen from Fig.5 and Table 1: The waveform is smooth after denoising by the soft threshold function while the accuracy of signal reconstruction is poor, and There are some oscillation points by the soft threshold function. According to the two indicators of SNR and MSE, the denoising effect of the proposed method is better than the traditional method, which can overcome the constant deviation effectively between the estimated value and the real value. It can also suppress the signal concussion phenomenon effectively in the hard threshold denoising method. In addition, according to the comparison results of LREPV, the local peak value of the signal can be better preserved and the same as preserving the local singularity of the signal, resulting in enhanced details of the signal preferably.

Table 1. Three threshold function denoising indicators

\begin{tabular}{cccc}
\hline & Hard threshold & Soft threshold & Threshold method proposed in this paper \\
\hline Signal-to-noise ratio $(\mathrm{dB})$ & 20.5667 & 19.8520 & 23.3172 \\
Root mean square error & 0.02134 & 0.03546 & 0.01827 \\
Local peak relative error $(\%)$ & 3.035 & 3.525 & 1.754 \\
\hline
\end{tabular}




\section{CONCLUSION}

In order to satisfy the need of denoising of target echo signal, a new threshold function with parameters is proposed in this paper, which is continuously derivable. Further, the optimal threshold in the sense of minimum mean square error is obtained by using the Stein unbiased risk estimate. The echo signal is denoised by

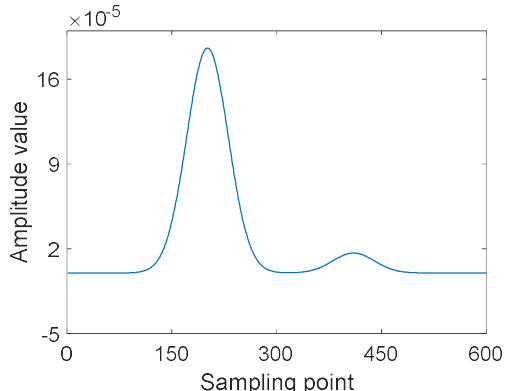

(a) Raw data

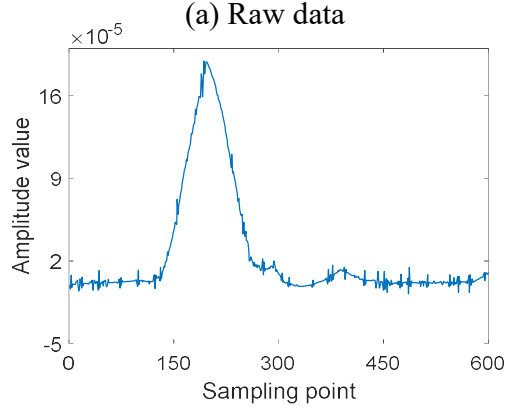

(d) Soft threshold denoising

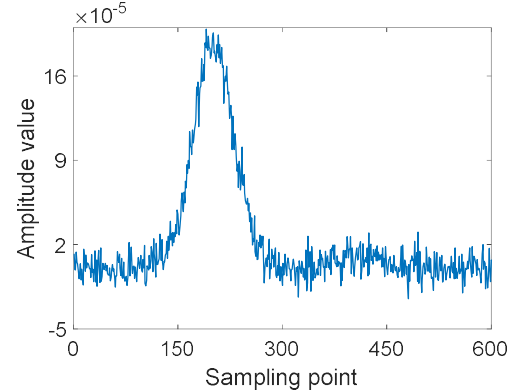

(b) Noise added data

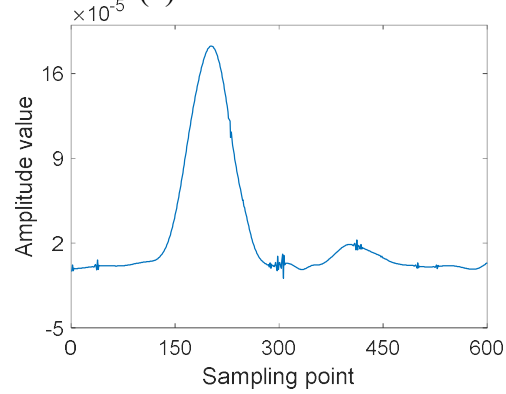

(e) Proposed method the proposed method under the expectation to preserve the singularity of the noisy signal while de-noising. Compared with the traditional hard threshold method and the soft threshold method, simulation experiment confirms the denoising efficacy of the proposed method that results in enhanced SNR and reduced MSE. The details of the original signal can be better preserved, and the signal denoising recovery ability is improved.

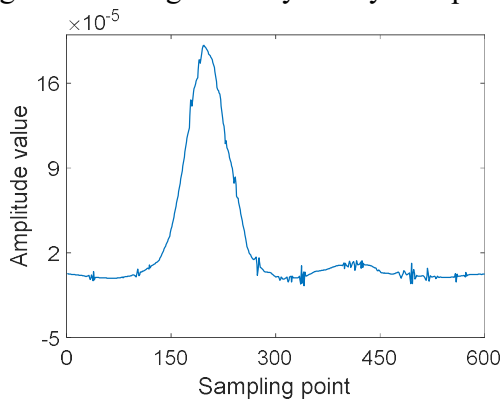

(c) Hard threshold denoising

Figure 5. Comparison of three threshold functions for denoising the echo signals denoising

\section{ACKNOWLEDGEMENTS}

This paper is financially supported by the National Natural Science of China under Grant numbers 41431179,41961065; Guangxi Innovative Development Grand Grant under the grant numbers GuikeAA18118038, GuikeAA18242048; the National Key Research and Development Program of China under Grant number 2016YFB0502501 and the BaGuiScholars program of Guangxi (Guoqing Zhou).

\section{REFERENCES}

Abdallah H., Baghdadi N., Bailly J S., Pastol Y., 2012. Wa-LiD: A New LiDAR Simulator for Waters. IEEE Geoscience and Remote Sensing Letters, 9(4):0-748.

Atto A M., Pastor D., Mercier G., 2011. Wavelet shrinkage: unification of basic thresholding functions and thresholds. Signal Image \& Video Processing, 5(1):11-28.

Beenamol M., Prabavathy S., Mohanalin J., 2012. Wavelet based seismic signal de-noising using Shannon and Tsallis entropy. Computers \& Mathematics with Applications, 64(11):35803593.

Deng G., Tay D B H., Marusic S., 2007, A signal denoising algorithm based on overcomplete wavelet representations and Gaussian models. Signal Processing, 87(5):866-876.

Donoho D L., 1995. De-noising by soft-thresholding. IEEE Transactions on Information Theory, 41(3):613-627.

Frei M G., Osorio I., 2007. Intrinsic time-scale decomposition: time-frequency-energy analysis and real-time filtering of non- stationary signals. Proceedings of the Royal Society A: Mathematical, Physical and Engineering Sciences, 463(2078):321-342.

Jansen M., Malfait M., Bultheel A., 1997. Generalized cross validation for wavelet thresholding. IEEE Trans Signal Process, 56(1):33-44.

Li J., Cheng C., Jiang T., 2012. Wavelet De-noising of Partial Discharge Signals Based on Genetic Adaptive Threshold Estimation. IEEE Transactions on Dielectrics and Electrical Insulation, 19(2):543-549.

Kim S C., Kang T J., 2006. Automated defect detection system using wavelet packet frame and Gaussian mixture model. Journal of the Optical Society of America Optics Image Science \& Vision, 23(11):2690-701.

Madeiro J P V., Cortez P C., Oliveira F I., Siqueira R S., 2007. A new approach to QRS segmentation based on wavelet bases and adaptive threshold technique. Medical Engineering \& Physics, 29(1):26-37.

Mallat S., Hwang W L., 1992. Singularity detection and processing with wavelets. IEEE Transactions on Information Theory, 38(2):617-643.

Nason G P., 1996. Wavelet Shrinkage Using Cross-Validation. Journal of the Royal Statistical Society. Series B(Methodological), 58(2):463-479.

Nasri M., Nezamabadi-Pour H., 2009. Image denoising in the wavelet domain using a new adaptive thresholding function.Neurocomputing, 72(4-6):1012-1025. 
Quan P., Dai G., Zhang H., Lei Z., 1998. Threshold selection method for hard-threshold filter algorithm. Acta Electronica Sinica, 26(1):115-118.

Quan P., Zhang L., Dai G., Zhang H., 1999. Two denoising methods by wavelet transform. IEEE Transactions on Signal Processing, 47(12):3401-3406.

Quan P., 2007. Wavelet Filtering Method and Its Application. Journal of Electronics \& Information Technology, 29(1):236242.

Randhawa S K., Sunkaria R K., Puthooran E., 2018. Despeckling of ultrasound images using novel adaptive wavelet thresholding function. Multidimensional Systems and Signal Processing, 30(3):1545-1561.

Sanam T F., Shahnaz C., 2013. Noisy speech enhancement based on an adaptive threshold and a modified hard thresholding function in wavelet packet domain. Digital Signal Processing, 23(3):941-951.

Stefan W., Chen K., Guo H., Renaut R A., 2012. Wavelet-Based De-noising of Positron Emission Tomography Scans. Journal of Scientific Computing, 50(3):665-677.

Stein, Charles M., 1981. Estimation of the Mean of a Multivariate Normal Distribution. The Annals of Statistics, 9(6):1135-1151.

Wu G., Wang C., Bao J., Chen Y., Hu Y., 2014. A Wavelet Threshold De-noising Algorithm Based on Adaptive Threshold Function. Journal of Electronics \& Information Technology, 36(6):1340-1347

Xu Q., 2002. Blue-Green LiDAR Ocean Survey. National Defence Industry Press.

Zhou G., 2007. LIDAR Data and Aerial Imagery Fusion, Remote Sensing of Impervious Surfaces, pp. 251-268, ISBN: 1-42004374-9.

Zhou G., Zhou X., Yang J., Tao Y., Nong X., Baysal O, 2015a. Flash Lidar Sensor Using Fiber-Coupled APDs. IEEE Sensors Journal, 15(9):4758-4768.

Zhou G., Chenxi Wang, Mingyan Li, Yuefeng Wang, Siqi Ye, Caiyun Han, 2015b. Advances and perspectives in bathymetry by airborne LiDAR, International Conference on Intelligent Earth Observing \& Applications, 0277-786X, Vol. 9808, 23-24, Guilin, China.

Zhou G., Zhou X., 2018. Principle, Technology and Applications for Array LiDAR Imager (Chinese), Wuhan Univ. Press, ISBN: 978-7-307-19683-4. 